\title{
Comment on "Maternal Satisfaction on Delivery Service and Its Associated Factors among Mothers Who Gave Birth in Public Health Facilities of Debre Markos Town, Northwest Ethiopia"
}

\author{
Siddharudha Shivalli \\ Department of Community Medicine, Yenepoya Medical College, Yenepoya University, Mangalore, Karnataka 575018, India \\ Correspondence should be addressed to Siddharudha Shivalli; shivalli.bhu@gmail.com \\ Received 19 August 2015; Accepted 31 August 2015 \\ Academic Editor: Mittal Suneeta \\ Copyright (C) 2015 Siddharudha Shivalli. This is an open access article distributed under the Creative Commons Attribution License, \\ which permits unrestricted use, distribution, and reproduction in any medium, provided the original work is properly cited.
}

I read the paper titled "Maternal Satisfaction on Delivery Service and Its Associated Factors among Mothers Who Gave Birth in Public Health Facilities of Debre Markos Town, Northwest Ethiopia" by Bitew et al. [1], with a great interest. Authors' efforts are highly commendable. It provides valuable information for evidence based interventions to enhance the quality and efficiency of maternal healthcare services in the study area. However, the following issues and concerns need to be addressed.

As it was a community based study and systematic random sampling was done, the following should have been mentioned: How many mothers were assessed for eligibility in the study area and how many of them were eligible and consented for voluntary participation (i.e., response rate)? These would enhance the internal validity of the findings.

Although authors mention "knowledge on ANC services" as one of the independent variables, but respective findings are not mentioned in the results.

It is not clear whether all or only the significant independent variables on univariate analysis were included in regression model. Authors should have mentioned the crude OR for all the independent variables considered in this study. And adequacy of the applied binary logistic regression model is not mentioned. Failure to do so may lead to misleading or incorrect inferences.

I feel that the following independent variables should have also been studied as they may influence the subjective feeling of satisfaction: predominant source of ANC (private or public health sector), gender of the baby, counseling, and level of birth preparedness and complication readiness.

Since satisfaction is very subjective (also quoted by the author) [2], top of mind response/s of the study participants as a feedback to improve the quality of healthcare services would have helped to fix the priorities.

\section{Conflict of Interests}

The author declares that there is no conflict of interests about this publication.

\section{References}

[1] K. Bitew, M. Ayichiluhm, and K. Yimam, "Maternal satisfaction on delivery service and its associated factors among mothers who gave birth in public health facilities of Debre Markos Town, Northwest Ethiopia," BioMed Research International, vol. 2015, 6 pages, 2015.

[2] J. H. Larrabee and L. V. Bolden, "Defining patient-perceived quality of nursing care," Journal of Nursing Care Quality, vol. 16, no. 1, pp. 34-60, 74-75, 2001. 


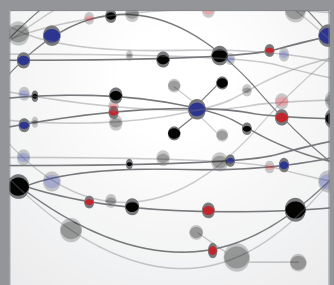

The Scientific World Journal
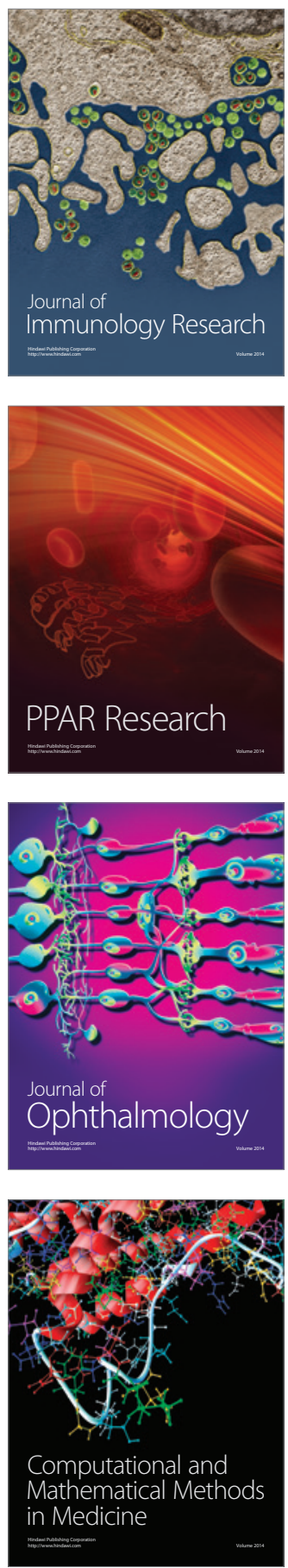

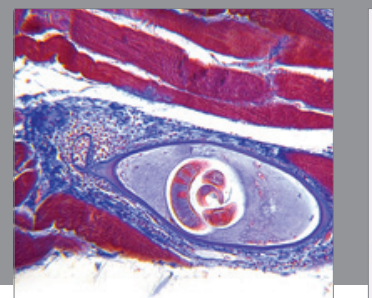

Gastroenterology

Research and Practice
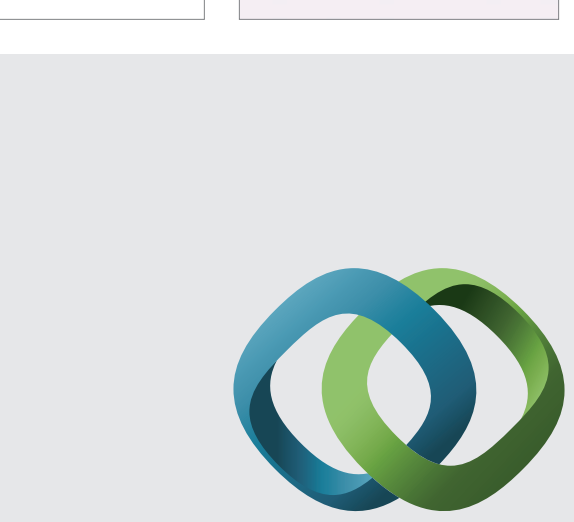

\section{Hindawi}

Submit your manuscripts at

http://www.hindawi.com
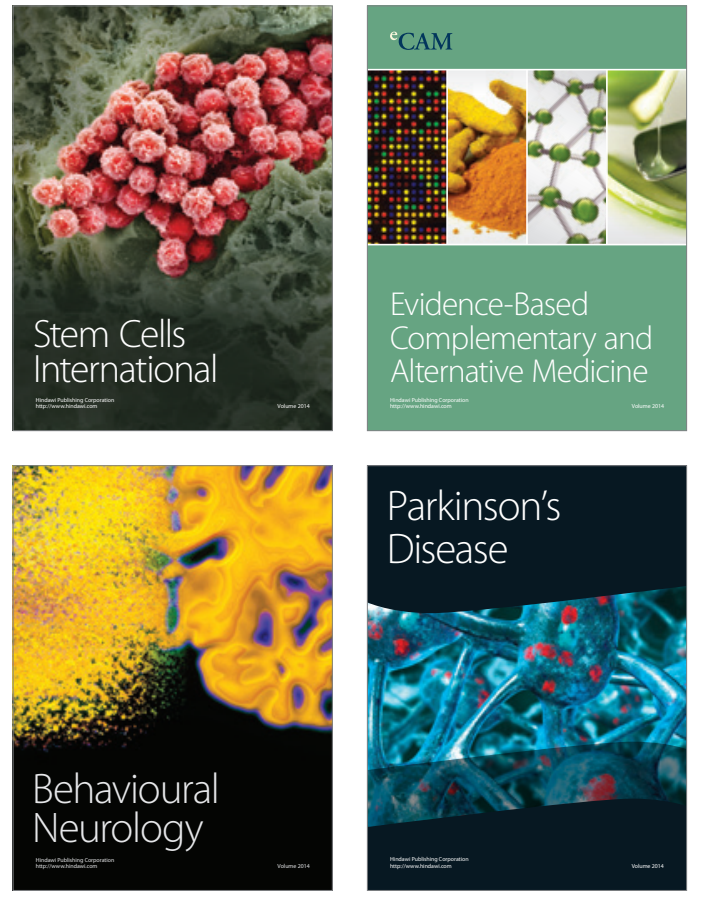
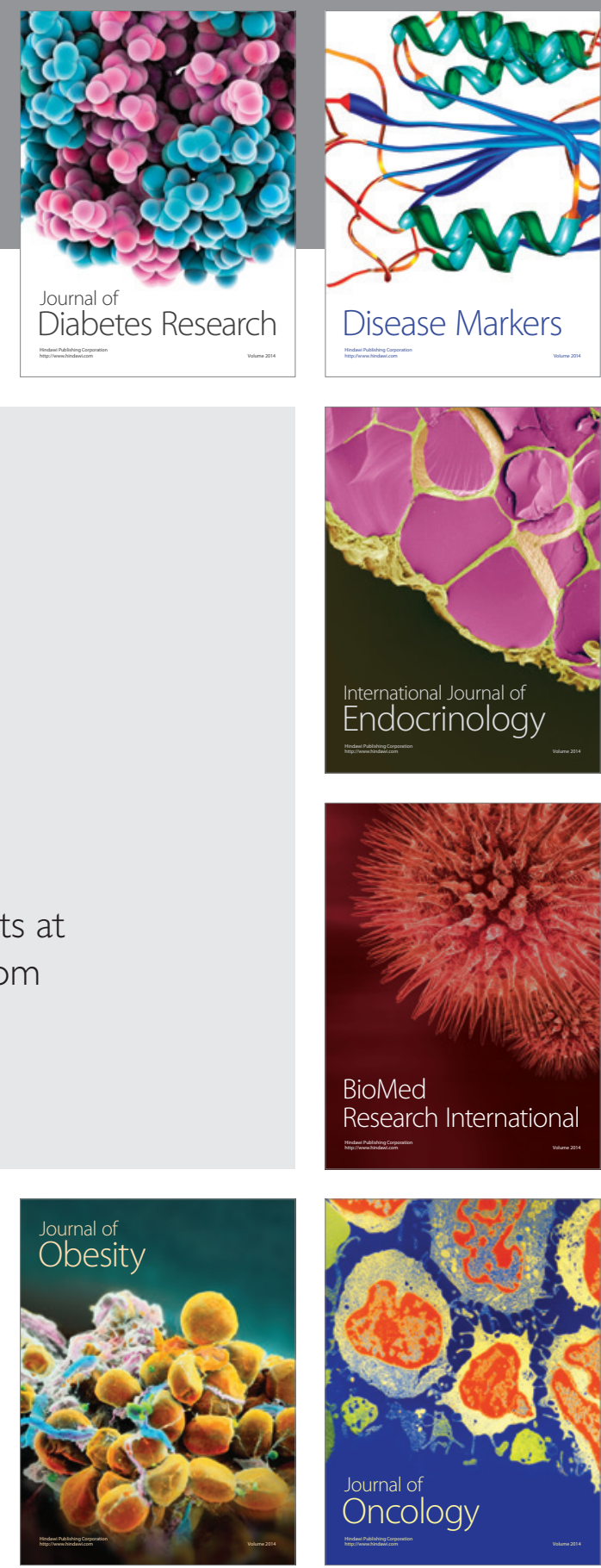

Disease Markers
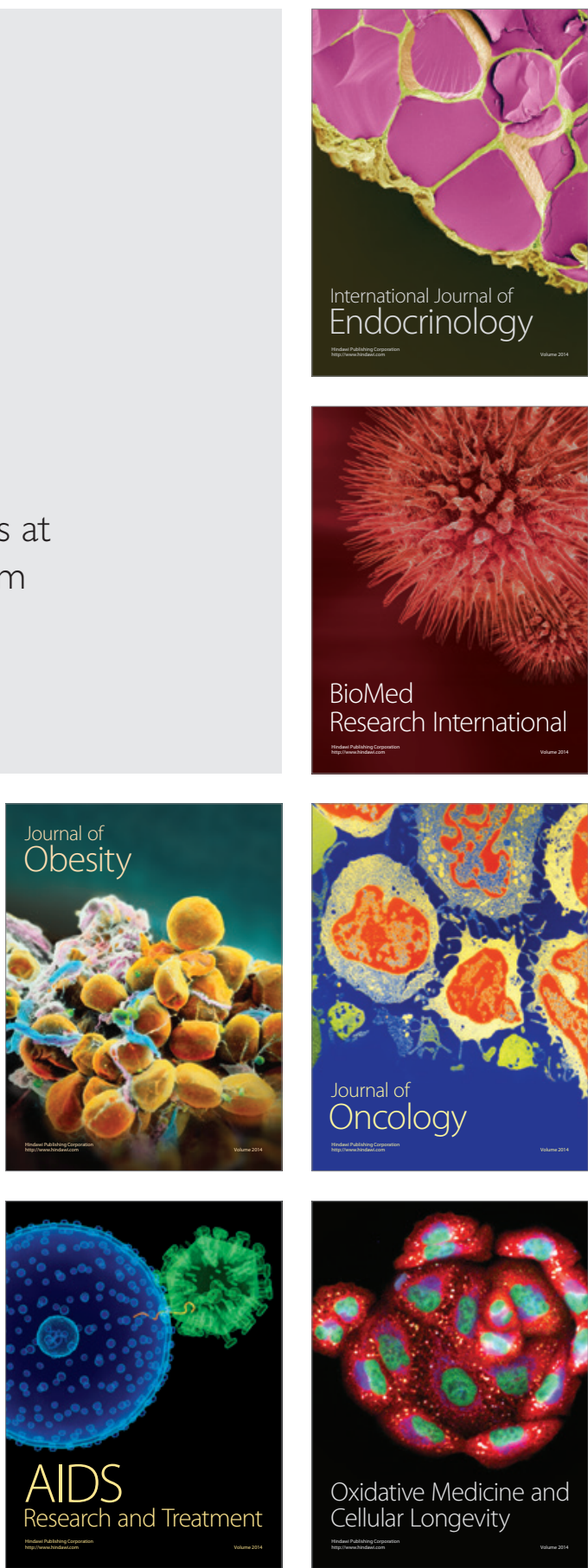